\title{
On the Findings of Stroke-prone SHR
}

\author{
Shigeki Takahashi, Yasuna HamaI, and Kozo OKamoto
}

The stroke-prone spontaneously hypertensive rats (SHRSP) develop severe hypertension rapidly and their incidence of strokes are constantly over $80 \%$. The correlation of the incidence of strokes and fundus findings has been reported by many investigators. But fundus findings of SHRSP were reported only by Dr. Yamori. In this paper we will report fundus finding of SHRSP and refer to the incidence of strokes.

\section{Methods:}

The fundus of 82 SHRSP at various ages were observed by an ophthalmoscope, without anesthetising the animals, with pupils dilated by Mydrin P (Tropicamidephenylephrine hydrochloride). The fundus camera (RCII Kowa Co Japan) was used for the photographs.

\section{Results :}

Several differences were noted between the retinal arterioles of SHRSP and those of SHR. That is, remarkably the tortuosity of retinal arterioles of SHRSP was observed in all stages. As the blood pressure elevated, narrowing of the retinal arterioles first appeared and then caliber irregularities followed. Most of the caliber irregularities changed into so-called aneurysma-like or rosary-like dilatation of arteriolar walls. On the other hand, in the hypertensive stage of SHRSP the optic discs became edematous (17 out of 82 cases). These findings became more manifest in the post-hypertensive stage. But the opacity and white patche of retina were not observed in any of the hypertensive stages. In the fundus of normotensive Wistar Kyoto no pathological changes were observed.

\section{Discussion:}

Authors previous reports described the fundus changes of SHR. According to this report, the retinal arterioles became narrower and caliber irregularities occurred with the elevation of blood pressure. In the fundus of SHRSP, narrowing and caliber irregularities of retinal arterioles increased and were more severe than those of SHR. Moreover, tortuosity of retinal arterioles of SHRSP was characteristic. Those tortuosity of retinal arterioles of SHRSP was thought to be related to the incidence of strokes.

\section{Summary :}

The fundus of 82 SHRSP were observed and all of them showed the narrowing, caliber irregularity of retinal arterioles and optic disc edema. Moreover, tortuosity of retinal arterioles was characteristic.

From the Department of Ophthalmology, Yamagata University School of Medicine, Yamagata; Department of Pathology, Kinki University School of Medicine, Osaka-fu. 